\title{
3D printer filament length monitor
}

\author{
Sharmila Borah \\ Dept. of CSE, BSAITM, Faridabad, India
}

Email address:

Sharmilaborah@gmail.com

To cite this article:

Sharmila Borah. 3D Printer Filament Length Monitor. International Journal of Science, Technology and Society.

Vol. 2, No. 5, 2014, pp. 129-132. doi: 10.11648/j.ijsts.20140205.16

\begin{abstract}
There have been many technological innovations. But only selective have been as revolutionary as 3D printer. It enables consumers to follow their own acquisition strategy. 3D printers has not only immersed into industrial design, footwear, engineering and construction, education but to medical application also. Although 3D printer is a great technological innovation, there still lays many problem, while operating with it. This paper focuses on the problem which is caused by 3D Printer filament. Filament is a very important and expensive part of 3D Printer. The filament is to be use carefully while printing an object in 3D Printer. This Paper will focuses on 3D Printer filament length monitor, which will update the user with the length of the filament, while printing and hence reduce the waste of filament by $90 \%$.
\end{abstract}

Keywords: 3D Printer, Filament

\section{Introduction}

3D printers have the capacity to make three-dimensional solid objects from digital designs. 3D printing is also known as additive manufacturing, as $3 \mathrm{D}$ printing is achieved by additive process, through additive process an object is created by layer down successive layers of materials.

Charles Hull invented a printing process called Stereolithography, in 1984 [1]. Through stereolithography digital data can be turned into tangible 3-dimensional objects. Charles Hull created the first stereolithography apparatus, which created complex 3D objects from photopolymer, though it was imperfect, but was the first step to the future of 3D printing. In 2011, Dr. Adrian Bowyer founded an open source 3D printers named as Reprap[2].

Today many 3D printers have immersed into the market such as Reprap Prusa i2, Reprap Prusa i3, Flashforge, Velleman, Printrbot and many more. Many of the 3D printers have open source models, and there are many open source firmwares, available for 3D printers such as marlin, sprinter and many more. So it has become very easy to manufacture the 3D printer now. That's the reason, today 3D have transformed almost all the fields.

People are giving a 3D shape to their imagination, with the help of the 3D Printer. To shape one imagination, filaments play an important role in that, it's because of different filament only, one can think of making different types of objects according to their needs and specifications.

Filaments are available in many varieties, and each filament has different properties. Filament performance is based on properties such as toughness, durability and moisture resistant. The most common filament, that are being use by 3D printers are PLA (Polylactic acid) and ABS (Acrylonitrile butadiene styrene). ABS has high strength and is malleable at 250 degrees Celsius, whereas PLA is brittle and shiny in nature and malleable at 60 degree Celsius only [3] [4]. Hence ABS can be use in printing parts for engineering design, whereas PLA can be use in printing jewellery parts, so according to the requirement filaments are to be chosen accordingly.

No doubt filaments are expensive and in many countries like India, Bangladesh, and Srilanka, Nepal and Pakistan, the $3 \mathrm{D}$ printing filament is not easily available. So filaments are to be use very carefully not only in the above mentioned countries but in all countries, since the filaments like PLA, Polycarbonate, and Nylon cannot be recycled. In this paper filament length monitor will be discussed through which the wasting of filament can be saved to $90 \%$.

\section{Methodology}

\subsection{Overview}

Whenever, we require printing an object through 3D printer, first we need to install the spool of filament into it 
and then power on the $3 \mathrm{D}$ printer, so that $3 \mathrm{D}$ printer can print as many as objects one needs. Many times 3D printer stops in between the printing, this happens because of many reasons, such as power cut off and shortage of filaments, which leads to wastage of filament.

The power cut off issue can be solve by installing UPS between main power and 3D printer and the shortage of filament issue can be handled by Filament length monitor.

Filament Length Monitor will keep monitoring the length of the filament being used by the 3D printer and hence will keep updating the remaining length of the filament. According to which we can make a judgment in selecting a suitable object which can get printed completely without any shortage of filaments.

\section{Design and Development}

The device Filament length monitor is based on the Arduino Uno board. The other main components, which are being used in filament length monitor, are:

1) Circular Bearing and Circular bearing Mount:

The role of circular bearing in the filament length monitor is to pass the filament and accordingly update the filament being used or the remaining filament available for printing.

Circular bearing of $4 \mathrm{~mm}$ diameter is used in the device, which is mounted on the circular bearing mount as shown in the figure1 given below.

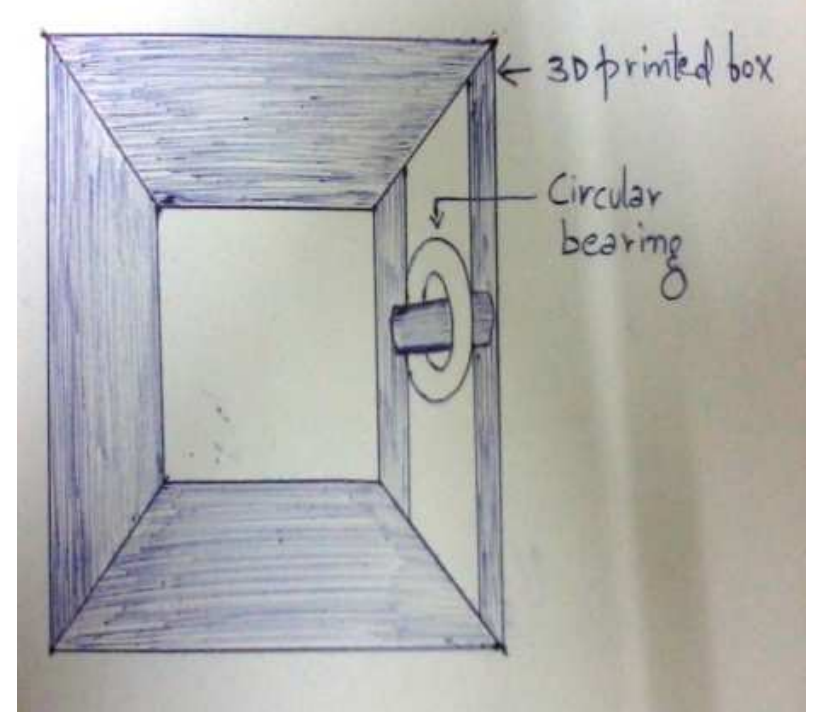

Figure 1. Circular bearing mount

Circular bearing mount is basically a 3D printed part, which act as a support to the circular bearing and to the screw. On one side of the circular bearing a limit switch is mounted. Which when will get pressed by a screw mounted on the opposite side of the bearing mount, will indicate the completion of one rotation and filament length will get updated. The screw and limit switch mounted on bearing mount is shown in the figure 2 as given below.

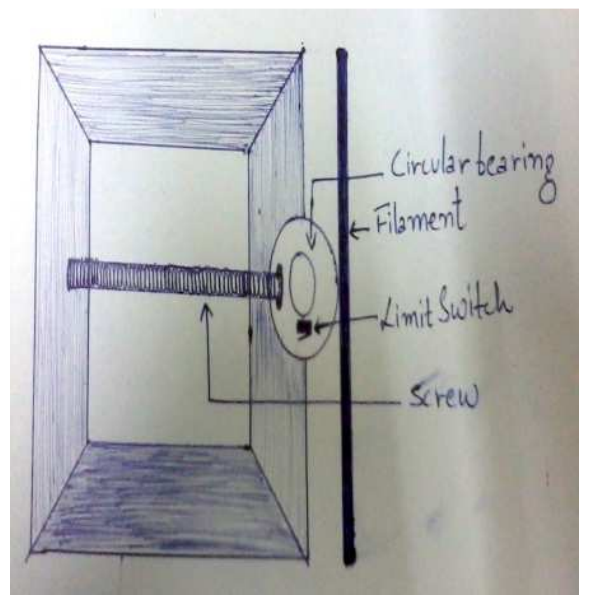

Figure 2. Circular bearing mount connecting to screw and limit switch

2) Limit switch and $\operatorname{LCD}(16 \times 2$ or $20 \times 4)$

The limit switch is of 3 pins will be mounted on one side of bearing, which when will get pressed by a screw will indicate the completion of one rotation. After each rotation the length of the filament will get updated on the LCD. The lcd and limit switch both are connected to the arduino Uno board as shown in the figure 3 given below.

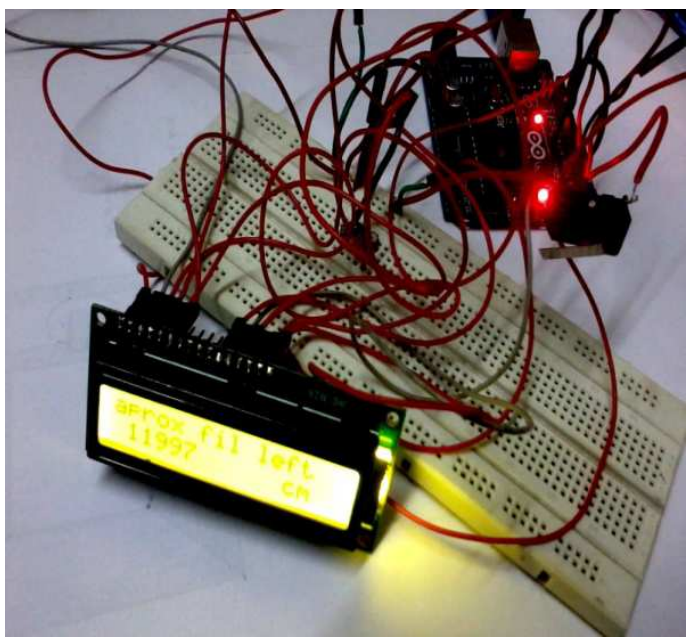

Figure 3. Arduino Uno connected to LCD and limit switch

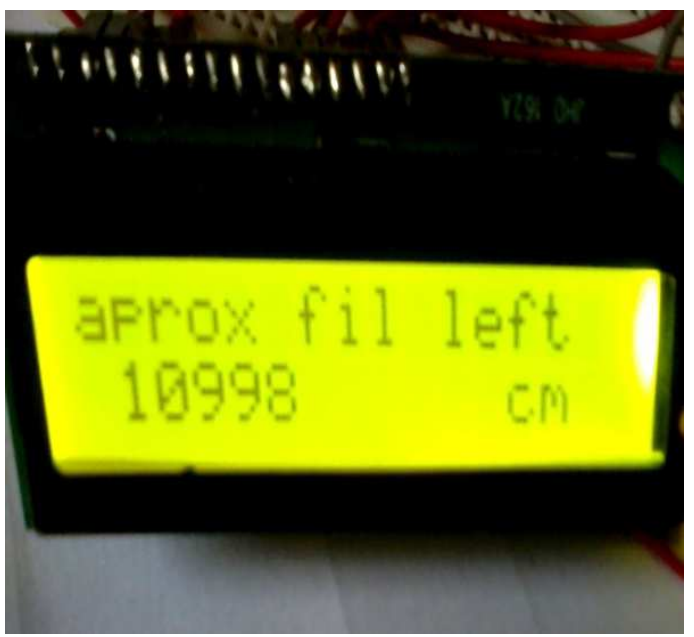

Figure 4. Closer view to the LCD of the filament length monitor 


\subsection{Procedure}

Different filament comes in different length with the spool. The filament diameter also affects the length of the filament in a given spool, since there are two different diameters of every filament that are $1.75 \mathrm{~mm}$ and $3.00 \mathrm{~mm}$.

Whenever we get a filament its density, volume and weight are always given. So by taking given amount, we can calculate the approximate filament length by, using the formulas given below.

$$
\text { Radius }=\text { diameter } / 2
$$

Diameter can be $1.75 \mathrm{~mm}$ or $3.0 \mathrm{~mm}$ depending upon the diameter of the filament.

$$
\begin{gathered}
\text { Area }=3.1415 * \text { radius } * \text { radius } \\
\text { Length of filament }=\text { weight } / \text { Area*density }
\end{gathered}
$$

The length of the filament can be taken as a reference for further calculation for updating the filament available for printing.

The filament is made in contact to the circular bearing. So that when the filament will move for extruding, the circular bearing will also move along with the filament. When the circular bearing will complete one rotation (when switch will get pressed by screw, it indicates one rotation) by moving along with the filament, then the used filament can be calculated by the formula given below.

Circumference of bearing $=2 * 3.1415 *$ radius of bearing

In the hardware, it is mentioned that $4 \mathrm{~mm}$ diameter circular bearing is being used by the filament length monitor. So according to the calculation approximately $1 \mathrm{~cm}$ of filament should get extruded after each rotation. Now after each rotation $1 \mathrm{~cm}$ of filament can be deducing from the reference length of the filament, which we calculated using formula 3 . The deducted filament length will act as a reference for next rotation and the LCD will keep updating the approximate filament length which is left for printing.

\subsection{Software}

The software is made open source, so that the user can make the changes in the software according to their requirement. In the software the two most common filaments is taken into account that are PLA and ABS, although the user can add more filament types to it. The main part of the program is given below.

$$
/ / 1=3.0 \mathrm{~mm}
$$

$/ / 2=1.75 \mathrm{~mm}$

\#define size 2 // select the size according to the above table

$$
/ / 1=\text { PLA }
$$

$/ / 2=\mathrm{ABS}$

\#define filament $1 / /$ select the filament according to above table

int buttonPushCounter ; // counter for the number of button presses

int buttonState $=0 ; / /$ current state of the button int lastButtonState $=0$; // previous state of the button int filament_left; // current state of filament length void $\operatorname{setup}()\{$

lcd.begin(16,2); // LCD type can be $16 \times 2$ 0r $20 \times 4$

lcd.setCursor( $(0,0)$;

lcd.print("aprox filament left");

if $($ size $==1 \& \&$ filament $==1)$

\{

filament_left $=33000$;

//filament legth of $3.0 \mathrm{~mm}$ PLA

\}

if $($ size $==1 \& \&$ filament $==2)$

\{

filament_left $=40000$;

//filament length of $3.0 \mathrm{ABS}$

\}

if $($ size $==2 \& \&$ filament $==1)$

\{

filament left $=11000$;

//filament length of $1.75 \mathrm{~mm}$ PLA

\}

if $($ size $==2 \& \&$ filament $==2)$

\{

filament_left $=13000$;

//filament length of $1.75 \mathrm{~mm}$ ABS

\}

void $\operatorname{loop}()\{$

buttonState $=$ digitalRead(buttonPin);

if (buttonState != lastButtonState) \{

if (buttonState $==\mathrm{HIGH})\{$

buttonPushCounter++;

filament_left--;

In the above program the reference filament length is already given for PLA and ABS for $1 \mathrm{~kg}$ of weight, by following the standards. The filament lengths which have been referred are:

PLA

$1.75 \mathrm{~mm}$ filament length for $1 \mathrm{~kg}$ of spool: $330 \mathrm{~m}$

$3.00 \mathrm{~mm}$ filament length for $1 \mathrm{~kg}$ of spool: $1100 \mathrm{~m}$

ABS

$1.75 \mathrm{~mm}$ filament length for $1 \mathrm{~kg}$ spool: $400 \mathrm{~m}$

$3.00 \mathrm{~mm}$ filament length for $1 \mathrm{~kg}$ spool: $130 \mathrm{~m}$

The lengths of the filaments are taken in centimeter, in the program, and are taken as reference length of the filament. Now after completion of one rotation, $1 \mathrm{~cm}$ (according to above calculation using formula 4 . by taking radius of bearing as $2 \mathrm{~mm}$ ) of filament will get deduce from the reference length of the filament.

The user can make changes in the size and filaments and in the filament length, by using formula 3. The user can add more no. of filament types and according to that can add more conditions to the program given above. 


\section{Results}

Filament length monitor approximately saves the filament, by updating with the filament length available for printing and according to which one can chose a suitable object to print.

It can be observed that the filament length monitor is not $100 \%$ accurate. But it can be made approximate by using the formulas given above. It can save $90 \%$ of wastage of filament, which takes places due to shortage of filament.

\section{Conclusion \& Future Scope}

Filament length monitor can successfully saves the wastage of filaments by $90 \%$. The filament length monitor can be made smarter by adding a memory card to it, through which it can, saves the amount of filament left in the memory card for further use.

\section{References}

[1] Charles W. Hull, Apparatus for production of three dimensional object by stereolithography, United States Patent, Mar 11, 1986.
[2] Rhys Jones, Patrick Haufe, Ed Sella, Peiman Iravani, Vik Ollicer, Chris Palmer, Adrain Bowyer, RepRap- The replicating rapid prototype, Robotica Volume 29, pp.177191(2011).

[3] ABS, Stratasys Inc,2007.

[4] Material safety datasheet, American Polymer standards Corporation, 2011.

[5] Dr. Rania F mohamed and Dr. Abeer S. Mahmoud, Emphasizing the advantage of 3D printing technology in packaging design development and production in local industries, International Design Journal, Volume 1 issue 1.

[6] 3D Printing a patent overview, Intellectual Property office, november 2013.

[7] Industry Journals, Siemens, January 2013.

[8] The potential of 3D printing, METI journal.

[9] Mathelde Berchon, Christina Wainikka, Waldemar Ingdahl, Per Stromback, 3D printing technology and beyond, november 2013. 